Check for updates

Cite this: RSC Adv., 2018, 8, 28581

Received 16th June 2018

Accepted 4th August 2018

DOI: $10.1039 / \mathrm{c} 8 \mathrm{ra0} 5170 \mathrm{~d}$

rsc.li/rsc-advances

\section{Continuous and rapid fabrication of photochromic fibers by facilely coating tungsten oxide/polyvinyl alcohol composites $\uparrow$}

\author{
Zhongwen Ling, ${ }^{\text {ab }}$ Kang Liu, ${ }^{a}$ Qi Zou, ${ }^{a}$ Qingsong Li, (D) a Ke-Qin Zhang, (D) a \\ Zheng Cui, ${ }^{b}$ Wei Yuan (D*b and Yuqing Liu*a
}

\begin{abstract}
Photochromic fibers have attracted great attention due to their wide use in areas of military camouflage, safety warnings, anti-counterfeiting, entertainment, etc. Compared with photochromic organic materials, inorganic photochromic tungsten trioxide $\left(\mathrm{WO}_{3}\right)$ materials have been extensively studied, because of their good stability and cost efficiency. In this work, we report the continuous fabrication of photochromic fibers in a simple and low-cost way by dip-coating $\mathrm{WO}_{3} /$ PVA composites. The prepared photochromic fibers show fast and reversible color switch from light yellow to dark blue upon UV irradiation and infrared heating treatment. The obtained photochromic fibers can be produced on a large scale and be woven into various patterns with good mechanical strength and washability, showing great potential in developing photochromic textiles.
\end{abstract}

\section{Introduction}

Modern clothing is currently moving towards more and more intelligent applications with the addition of some high-end functionalities, such as physiological signal acquisition, ${ }^{\mathbf{1}}$ performance monitoring, ${ }^{2}$ flexible electronics and display, ${ }^{3}$ temperature control, ${ }^{4}$ energy storage, ${ }^{5}$ etc. However, most of the current so-called "smart clothing" is made of technologies being integrated into common garments rather than made of truly functional fibers. Nevertheless, many fibrous devices have been extensively developed in the past few years owing to their unique one dimensional structure with the ability to perfectly implant into textiles or clothes and with promising applications in portable and wearable devices, such as solar cells, ${ }^{6}$ supercapacitors, ${ }^{7}$ color variation, ${ }^{8}$ lithium ion batteries, ${ }^{9}$

${ }^{a}$ National Engineering Laboratory for Modern Silk, College of Textile and Clothing Engineering, Soochow University, Suzhou 215123, China. E-mail: liuyuqing@suda. edu.cn

${ }^{b}$ Printable Electronics Research Centre, Suzhou Institute of Nanotech and Nanobionics, Chinese Academy of Sciences, Suzhou 215123, China.E-mail: wyuan2014@ sinano.ac.cn

$\dagger$ Electronic supplementary information (ESI) available: The original pictures of $\mathrm{WO}_{3}$ nanorods powder; UV-vis diffuse reflectance spectra of the $\mathrm{WO}_{3}$ nanorods powder with different infrared heating time; the pictures of $\mathrm{WO}_{3} / \mathrm{PVA}$ composite films with different $\mathrm{WO}_{3}$ nanorods contents; the pictures of three kinds of coating solutions with different $\mathrm{WO}_{3}$ content after mixing and standing for $2 \mathrm{~h}$ at room temperature; the coating thickness as function of fiber drawing speed and SEM images of the surface of coated fiber with different drawing speeds; UV-vis diffuse reflectance spectra of one single photochromic fiber with different UV irradiation time; schematic diagram of the experimental process for studying the discoloration process of composite fiber by using various oxidation methods. See DOI: 10.1039/c8ra05170d electroluminescence, ${ }^{\mathbf{1 0}}$ etc. These functional fibers represent a significant strategy towards the development of intelligent textiles.

Among the various functional fibers, smart color-changing fibers have become a hotspot for their potential applications, such as wearable displays, visual sensors, military camouflage and so on. ${ }^{\mathbf{1 1 - 1 5}}$ So far, the existing smart chromatic fibers can change their colors upon encountering diverse stimuli like temperature, ${ }^{\mathbf{1 6}}$ ray radiation, ${ }^{17}$ specific solvents and gas, ${ }^{\mathbf{1 8 , 1 9}}$ stress tension ${ }^{20}$ and electricity. ${ }^{21-23}$ Although electricity was usually thought to be an active and controllable approach to trigger the color change, the fabrication of electrochromic or electrothermal chromatic fibers is not easy due to their complex device structure with solid and liquid phase components. The multi-layer coating process, including conductive layer, photocatalytic layer, thermochromic layer and encapsulation layer, leads to difficulties in continuous mass production. Power supply is essential for these chromatic fibers and current pass through the whole fibers, which raise obvious safety considerations for wearable applications. Compared with electrochromic devices, photochromic applications are free of complex device configurations and electric supply. Just upon photoirradiation, coloration occurs. ${ }^{24,25}$

Therefore, in this work, we designed a photochromic fiber with simple core-shell structure. Of the photochromic materials studied so far, tungsten trioxide $\left(\mathrm{WO}_{3}\right)$ has become one of the most extensively studied candidates. Integrating $\mathrm{WO}_{3}$ nanomaterials into a polymer matrix is a feasible method for preparing composite materials with controllable photochromic properties. Several groups have studied the photochromism of the inorganic/organic composite photochromic materials. ${ }^{26-33}$ 
Kuboyama et $a .^{26}$ prepared polyethylene glycol (PEG) $/ \mathrm{WO}_{3}$ hybrids and studied their photochromic properties. The photochromism of $\mathrm{WO}_{3}$ was dramatically enhanced in the presence of PEG. Yano et $a .^{27}$ produced poly(vinyl alcohol) (PVA) $/ \mathrm{WO}_{3}$ hybrid by using ion exchange process of $\mathrm{Na}_{2} \mathrm{WO}_{4}$ $\cdot 2 \mathrm{H}_{2} \mathrm{O}$. Good mechanical and photochromic properties were presented by the $\mathrm{PVA} / \mathrm{WO}_{3}$ hybrids. Chen et al. ${ }^{28}$ successfully prepared $\mathrm{WO}_{3}-\mathrm{TiO}_{2}-\mathrm{ZnO}$ ternary composite films by a sol-gel method using PEG-400 as an organic additive. The addition of PEG-400 helped to improve the photochromism of composite film. Yamazaki et al. ${ }^{29}$ fabricated photochromic film by incorporating $\mathrm{WO}_{3}$ nanoparticles in methylcellulose matrix with addition of little amount agents. The biodegradable composite film had good photochromic properties with high contrast of the color change before and after UV irradiation. Recently, Zhou et $a{ }^{30}$ prepared photochromic inks by dispersing the $\mathrm{WO}_{3}$ nanoparticles into aqueous PVP solution, and casted the photochromic films on flexible substrate. The composite film exhibited fast photochromic switching rate and good reversibility. Although these composite materials based $\mathrm{WO}_{3}$ and polymers have been received much attention, and have been used in various applications, such as, photochromic films, ${ }^{30}$ bio-electronic devices, ${ }^{31}$ rewritable media $^{32}$ et al., these researches are mainly focused on photochromic composite film, while the photochromic fiber based on $\mathrm{WO}_{3}$ is rarely report. Recently, some hybrid $\mathrm{WO}_{3}$ /polymer nanofibers have been prepared via electrospinning, ${ }^{34-37}$ while these electrospun nanofibers are all applied in form of nonwoven fabric rather than the continuous monofilament because of low mechanical strength. Therefore, in this paper, the basic idea of this research is preparation of photochromic fiber with good mechanical strength by coating $\mathrm{WO}_{3} / \mathrm{PVA}$ composites. The produced functional fiber has good photochromic properties, and can be woven into fabric blending with ordinary fiber, which may apply in some special fields like military, firefighting etc.

Herein, a simple process was employed to prepare photochromic fiber based on $\mathrm{WO}_{3}$ nanomaterials. The first step was to prepare light-yellow $\mathrm{WO}_{3}$ nanorods by a one-step hydrothermal method. The $\mathrm{WO}_{3}$ nanorods were then uniformly dispersed into PVA solution by magnetic stirring, and finally coated on the surface of cotton fiber using a rapid and continuous dip-coating process. The $\mathrm{WO}_{3}$-based photochromic fiber exhibited fast photochromic performance, good reversible color change. The produced photochromic fiber also displayed good mechanical strength and stability, and could be woven into various patterns and fabrics. This study proposes a feasible strategy for large-scale preparation of photochromic fiber, and the produced photochromic fiber may have potential applications in the areas of military, firefighting et al.

\section{Experimental section}

\subsection{Materials}

Sodium tungstate dehydrate $\left(\mathrm{Na}_{2} \mathrm{WO}_{4} \cdot 2 \mathrm{H}_{2} \mathrm{O}\right)$ used in this study was supplied by shanghai Titan Scientific Co., Ltd. Potassium sulfate $\left(\mathrm{K}_{2} \mathrm{SO}_{4}\right)$ was purchased from Jiangsu Strong Functional Chemical Co., Ltd. Oxalic acid dihydrate $\left(\mathrm{C}_{2} \mathrm{H}_{2} \mathrm{O}_{4} \cdot 2 \mathrm{H}_{2} \mathrm{O}\right)$ was purchased from Admas Reagent Co., Ltd. Polyvinyl alcohol (PVA, 1799), hydrochloric acid and ethanol were purchased from Sinopharm Chemical Reagent Co., Ltd. Cotton fiber was purchased from the commercial market. All of the chemicals were used as received without further purification. Water used in all experiments was ultra-pure water.

\subsection{Synthesis of $\mathrm{WO}_{3}$ nanorods}

$\mathrm{WO}_{3}$ nanorods were synthetized as the following process. ${ }^{33} 4 \mathrm{~g}$ $\mathrm{Na}_{2} \mathrm{WO}_{4} \cdot 2 \mathrm{H}_{2} \mathrm{O}$ and $3.3 \mathrm{~g} \mathrm{C}_{2} \mathrm{H}_{2} \mathrm{O}_{4} \cdot 2 \mathrm{H}_{2} \mathrm{O}$ were dissolved in $100 \mathrm{ml}$ ultra-pure water to form a homogeneous solution. Next, the solution was acidified to a $\mathrm{PH}$ value of 1.68 by using $3 \mathrm{~mol} \mathrm{~L}^{-1}$ hydrochloric acid. And then $6 \mathrm{~g} \mathrm{~K}_{2} \mathrm{SO}_{4}$ was added into the solution with stirring $1 \mathrm{~h}$ to form a transparent and homogeneous precursor solution. Next, the prepared precursor solution was transferred into a Teflon-lined autoclave with a stainless steel shell. After reaction at $180{ }^{\circ} \mathrm{C}$ for $36 \mathrm{~h}$, the final naturally cooled products were collected by vacuum filtering and washed separately with ultra-pure water and ethanol to remove residual ions. Finally, the obtained products were dried at $60{ }^{\circ} \mathrm{C}$ in a vacuum oven.

\subsection{Preparation of composite solution of $\mathrm{WO}_{3}$ and PVA}

Firstly, $10 \mathrm{wt} \%$ transparent PVA solution was prepared by dissolving PVA in DI water at $85{ }^{\circ} \mathrm{C}$ under stirring for $4 \mathrm{~h}$. Then, the as-synthesized $\mathrm{WO}_{3}$ nanorods powders were added to the prepared PVA solution with three different $\mathrm{WO}_{3}$ solid contents: $1 \mathrm{wt} \%, 3 \mathrm{wt} \%, 5 \mathrm{wt} \%$. The mixtures were pre-stirred at $1000 \mathrm{rpm}$ for $10 \mathrm{~min}$, and then $1 \mathrm{wt} \%$ no-ionic surfactant (TX-100) related to amount of $\mathrm{WO}_{3}$ were added to improve dispersion of $\mathrm{WO}_{3}$ nanorods in PVA solution. ${ }^{38,39}$ Finally, the mixtures were agitated strongly using stirring bar at $1000 \mathrm{rpm}$ for $1 \mathrm{~h}$ to gain the homogeneous $\mathrm{WO}_{3} / \mathrm{PVA}$ gel solution.

\subsection{Continuous fabrication of photochromic fiber}

As shown in Fig. 1, the photochromic fiber was prepared continuously by three steps: (1) surface coating; (2) fast coagulation; (3) dry collection. The detailed process was described as follows: firstly, the homogeneous blend solution was placed in a $5 \mathrm{ml}$ lab-made conical vessel with aperture inner diameter of $0.8 \mathrm{~mm}$, which was fixed vertically on an iron stand. The cleaned and oxygen plasma ( $100 \mathrm{~W}, 5 \mathrm{~min})$ pre-treated cotton fiber, was drawn slowly at constant speed $\left(10 \mathrm{~mm} \mathrm{~min}^{-1}\right)$ through the blend solution by a computer controlled motor. Secondly, the coated fiber was immersed into alcohol bath subsequently to coagulate the $\mathrm{WO}_{3} / \mathrm{PVA}$ composite coating layer quickly. Finally, the cotton fiber with coagulated coating was dried at $70{ }^{\circ} \mathrm{C}$ with infrared lamp and collected using a stainless roller with diameter of $2 \mathrm{~cm}$.

\subsection{Characterization}

The transmission electron microscopy (TEM) images, high resolution transmission electron microscopy (HRTEM) images and the electron diffraction pattern were collected with a transmission electron microscope (FEI Tecnai G2 F20). The 


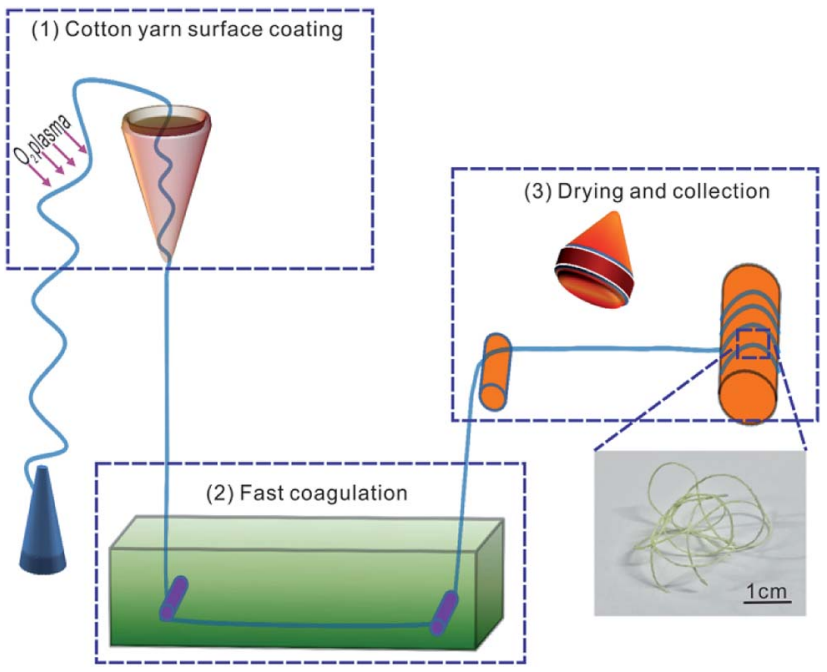

Fig. 1 Schematic illustration of the continuous fabrication process of photochromic fiber.

scanning electron microscopy (SEM) images, the selected area elemental analysis and the energy dispersive spectroscopy (EDX) were performed by a Field Emission-SEM system (Hitachi S-4800). X-ray diffraction (XRD) patterns were collected by a Philips X'Pert-Pro MRD diffractometer with a $\mathrm{Cu} \mathrm{K} \alpha$ radiation. X-ray photoelectron spectroscopy (XPS) was measured by a Kratos AXIS ULTRA DLD system. All the optical pictures were taken by Nikon D750 digital camera, and the photochromic coloration process was carried out by using a handle UV lamp with $254 \mathrm{~nm}$ emission. The UV-vis diffuse reflectance spectroscopies of solid $\mathrm{WO}_{3}$ samples and photochromic fiber samples were captured by Ideaoptics PG4000 spectrometer system.

\section{Results and discussion}

The morphology and microstructure of as-synthesized samples were shown in Fig. 1a, which indicates the diameter of obtained $\mathrm{WO}_{3}$ nanorods is about $500 \mathrm{~nm}$. Phase purity and crystallographic structure of the samples were determined by XRD. As shown in Fig. 2b, all the diffraction peaks of XRD patterns can be indexed to the hexagonal phase of $\mathrm{WO}_{3}$ (JCPDS no. 75-2187). The TEM image of Fig. $2 \mathrm{c}$ further indicates the rod-like microstructure of the synthesized $\mathrm{WO}_{3}$. The HR-TEM image shown in Fig. $2 \mathrm{~d}$ illustrates that the spacing between adjacent lattice planes is $0.38 \mathrm{~nm}$, which is indexed as (002) plane of hexagonal $\mathrm{WO}_{3}$ and in accordance with XRD data. All the above measured results indicate that the $\mathrm{WO}_{3}$ nanorods with hexagonal structure have been successfully produced.

The reversible photochromic process of $\mathrm{WO}_{3}$ nanorods was examined with UV irradiation for coloration and the infrared lamp heating treatment for bleaching. The UV irradiation was implemented using a $36 \mathrm{~W}$ commercial UV lamp, and the heat treatment was performed by a $50 \mathrm{~W}$ infrared lamp. Fig. 3a shows the gradually changing photographs of $\mathrm{WO}_{3}$ nanorods powder during UV coloration and heat bleaching cycle with various exposure times. Under UV irradiation process, the color of $\mathrm{WO}_{3}$

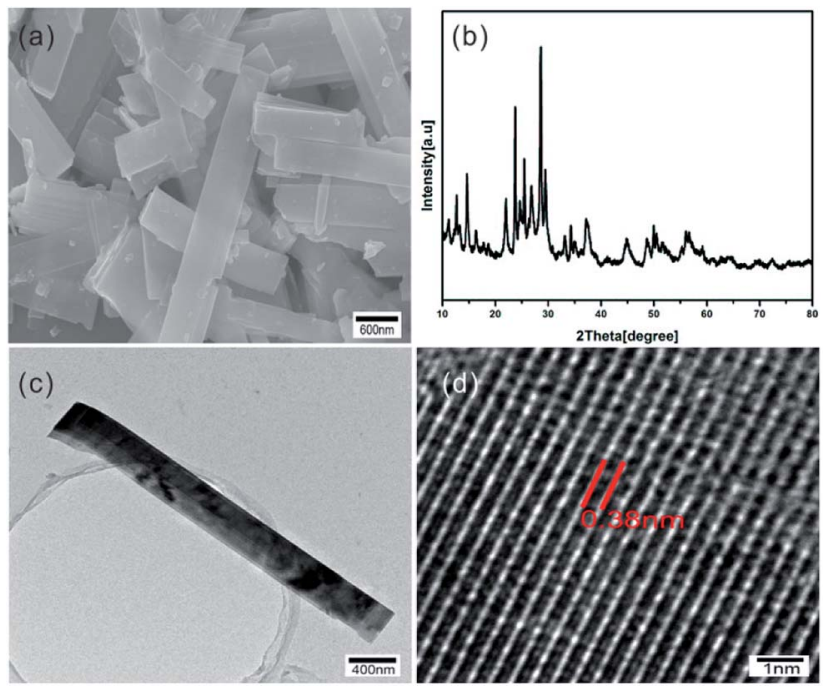

Fig. 2 (a) SEM image, (b) XRD pattern, (c) TEM image and (d) HR-TEM image of as-synthesized $\mathrm{WO}_{3}$ nanorods.
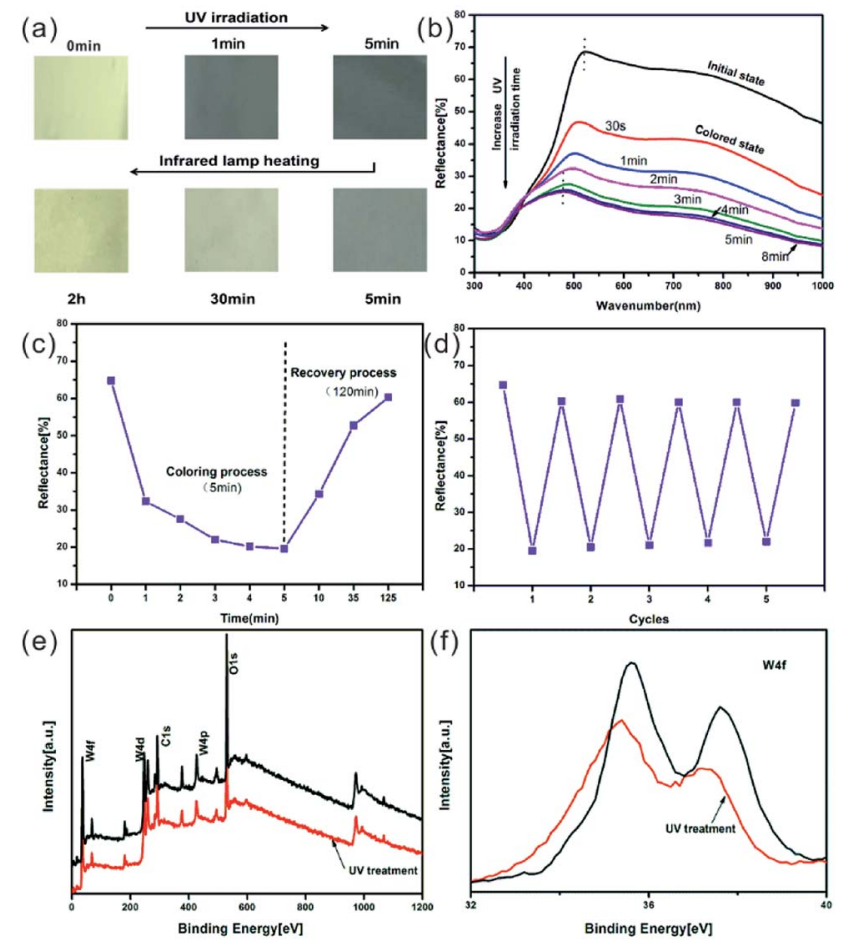

Fig. 3 (a) Photographs of the $\mathrm{WO}_{3}$ nanorods powder show gradual color change under UV irradiation and infrared heating, these pictures were cut and rebuilt from the original pictures as shown in Fig. S1; $\uparrow$ (b) $U V$-vis diffuse reflectance spectra of the $\mathrm{WO}_{3}$ nanorods powder with different UV irradiation time; (c) the reflectance intensity of the $\mathrm{WO}_{3}$ nanorods powder at $600 \mathrm{~nm}$ monitored for one coloration-bleaching cycle; (d) the reflectance intensity of the $\mathrm{WO}_{3}$ nanorods powder at $600 \mathrm{~nm}$ after $5 \mathrm{~min}$ UV irradiation (coloration) and $2 \mathrm{~h}$ infrared heating treatment (bleaching), monitored for continuous 5 cycles; (e) XPS survey spectra of $\mathrm{WO}_{3}$ nanorods powder before (black) and after (red) UV irradiation; (f) W 4 XPS for before (black) and after (red) UV irradiation states respectively. 
nanorods changed from initial pale yellow into dusty blue in one minute and gradually changed into dark blue color within 5 min. During the following infrared lamp heating treatment, the sample's color reversed back to its initial status within about 2 hours. The corresponding reflectance spectrums of color variation were measured as shown in Fig. $3 \mathrm{~b}$. The intensity of reflective peak dramatically decreased with UV irradiation, and finally stabilized at $27.6 \%$ (initial $67.9 \%$ ) after $5 \mathrm{~min}$. When extending the exposure time to $8 \mathrm{~min}$, the curve of spectrum almost unchanged. The reflectance spectrum can be recovered to its initial stage after $2 \mathrm{~h}$ heating treatment (Fig. $\mathrm{S} 2 \dagger$ ), which is in consistent with the discoloration of $\mathrm{WO}_{3}$ nanorods powder from blue to yellow (Fig. 3a). The intensities of spectrum curves at $600 \mathrm{~nm}$ were collected during one coloration-bleaching cycle as shown in Fig. 3c. In coloration process, the intensity decreased from $64.8 \%$ to $32.2 \%$ within 1 min and the coloration was saturated $(19.5 \%)$ with UV irradiation for $5 \mathrm{~min}$. In bleaching process, the intensity increased to $60.3 \%$ with $2 \mathrm{~h}$ heating treatment, which was slight lower than the initial value. If prolonging the thermal treatment time to $5 \mathrm{~h}$, the intensity of reflectance spectrum can fully recover. To evaluate the reversibility of color turning, the $\mathrm{WO}_{3}$ sample was processed with sequential UV irradiation for $5 \mathrm{~min}$ and heating treatment for $2 \mathrm{~h}$ repeatedly, and the corresponding reflectance intensities at $600 \mathrm{~nm}$ were recoded as 5 coloration-bleaching cycles as shown in Fig. 3d, which demonstrated a good reversibility of color switching.

XPS was employed to study the evolutional structural changes in the coloration process. The wide-scan XPS in Fig. 3e indicates that the $\mathrm{W}$ and $\mathrm{O}$ are the primary elements in $\mathrm{WO}_{3}$ nanorods without any other impurities. In order to further investigate binding state, the narrow-scan XPS spectra for W element was carried out before and after UV irradiation. In Fig. $3 \mathrm{f}$, the $\mathrm{W} 4 \mathrm{f}$ two peaks with binding energies of $35.6 \mathrm{eV}$ and $37.7 \mathrm{eV}$ corresponds to $\mathrm{W}^{6+}$ state in $\mathrm{WO}_{3}$ powder before $\mathrm{UV}$ treatment. And the energy separation between the main peaks of $\mathrm{W}_{4 \mathrm{f}_{7 / 2}}$ and $\mathrm{W} 4 \mathrm{f}_{5 / 2}$ was $2.1 \mathrm{eV}$, which further agreed with the tungsten in the $\mathrm{W}^{6+}$ valence state. After $\mathrm{UV}$ treatment, the $\mathrm{W} 4 \mathrm{f}$ shoulder peaks appear shift towards lower binding energy and the intensity also decreased. The newly formed shoulder peaks can be assigned as $\mathrm{W}^{5+}$ species, which were reduced upon UV irradiation.

The as-synthesized $\mathrm{WO}_{3}$ nanorods were mixed with PVA solution to form homogeneous blend coating solution as shown in Fig. 4a. With the addition of a non-ionic surfactant, the $\mathrm{WO}_{3}$ nanorods were uniformly dispersed in PVA solution with light yellow color. Three kinds of coating solutions were prepared with different weight content of $\mathrm{WO}_{3}$ nanorods $(1 \mathrm{wt} \%, 3 \mathrm{wt} \%$, $5 \mathrm{wt} \%)$. Fig. S3† shows these blend solutions all have good film forming abilities when they were spin-coated on glass substrate, and the films' color gradually deepens with the increase of $\mathrm{WO}_{3}$ content both before and after UV irradiation. However, as $\mathrm{WO}_{3}$ content increases, some precipitate occurs. Three kinds of coating solutions were observed after mixing and standing for $2 \mathrm{~h}$. A small amount precipitation appears in the blend solution with $5 \mathrm{wt} \% \mathrm{WO}_{3}$ content, but there's no obvious aggregation of $\mathrm{WO}_{3}$ in the blend solution with $3 \mathrm{wt} \%$ content, as shown in



(c) Untreatment

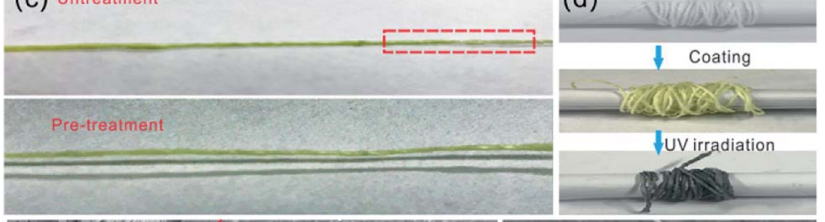

(d)

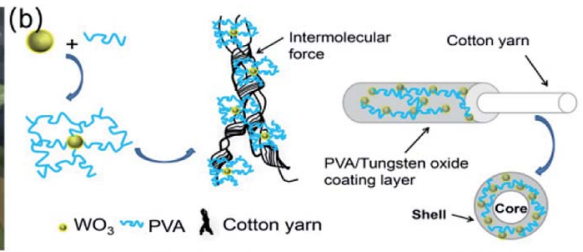

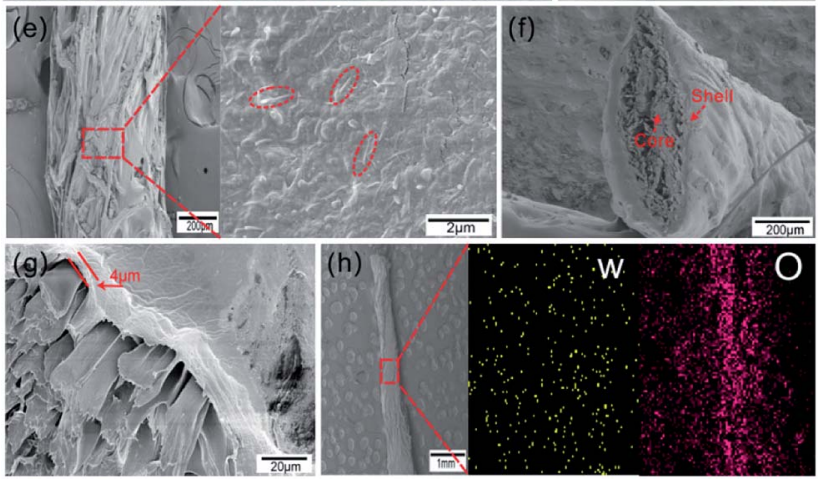

Fig. 4 (a) Picture of $\mathrm{WO}_{3} / \mathrm{PVA}$ blend coating solution with $\mathrm{WO}_{3}$ content of $3 \mathrm{wt} \%$; (b) the schematic diagram of the coating process; (c) pictures of photochromic fiber separately fabricated with pre-treated and untreated cotton fiber, the area marked with red dotted line shows the uneven coating layer; (d) pictures of pure cotton fiber (upper), photochromic fiber before (middle) and after (below) UV irradiation; (e) SEM images of photochromic fiber's surface with low and high magnification, the area marked with red dotted line indicates the embedded $\mathrm{WO}_{3}$ nanorods; (f) SEM image of photochromic fiber's cross-section; (g) SEM image of $\mathrm{WO}_{3} / \mathrm{PVA}$ coating layer's crosssection, the cotton fiber drawing speed was $10 \mathrm{~mm} \mathrm{~min}^{-1}$; (h) the elemental distribution maps of $\mathrm{W}$ and $\mathrm{O}$ elements of the composite coating layer.

Fig. S4. $\dagger$ Note that the precipitation can block the nozzle in the coating process, which may cause difficulties in continuous production. Therefore, the blend solution with $3 \mathrm{wt} \% \mathrm{WO}_{3}$ content was selected as coating solution for fabrication of photochromic fibers. The schematic diagram of Fig. $4 \mathrm{~b}$ shows the mechanism of coating process: $\mathrm{WO}_{3}$ nanorods were dispersed in PVA solution, when the cotton fiber was draw through the blend solution, $\mathrm{PVA} / \mathrm{WO}_{3}$ composite layer was uniformly coated on its surface with core-shell structure. $\mathrm{WO}_{3}$ nanorods were embedded in PVA polymer to form the functional coating layer with photochromic property. Note that cotton fiber must be pre-treated with $\mathrm{O}_{2}$ plasma to improve the hydrophilic property. Fig. 4c shows the picture of coated fibers with and without pre-treatment at the same drawing speed. It's obvious that the coating layer is not continuous and has bad binding force with the untreated cotton fiber. In contrast, the composite layer was continuously coated on the surface of pretreated cotton fiber with strong adhesion force due to hydrogen bonding. This manufacturing process is suitable for mass production, as indicated in Fig. $4 d$, the photochromic fiber in 
length of two meters was collected by a roller, and after UV irradiation, the light yellow color changed into dark blue.

The surface morphology of the photochromic fiber was observed by SEM, as shown in Fig. 4e. The images with low and high magnification indicate a dense layer of $\mathrm{PVA} / \mathrm{WO}_{3}$ composite was coated on the surface of cotton fiber, and the $\mathrm{WO}_{3}$ nanorods were uniformly embedded in the composite layer, as shown in the area marked with red dotted line. The cross section image in Fig. $4 \mathrm{f}$ shows the complete core-shell structure of the photochromic fiber, and the thickness of coating shell was about $4 \mu \mathrm{m}$ (Fig. $4 \mathrm{~g}$ ). Note that the coating thickness negatively correlate with the fiber drawing speed. In order to form continuous and uniform coating layer, various drawing speeds were tested and the detailed relationship between thickness and drawing speed was shown in Fig. S5.† Finally, the speed of $10 \mathrm{~mm} \mathrm{~min}{ }^{-1}$ was performed as the drawing speed of cotton fiber in the continuous process. The elemental distribution maps of $\mathrm{W}$ and $\mathrm{O}$ elements of the composite coating layer (Fig. 4h) show uniform distributions of the $\mathrm{WO}_{3}$ nanorods, which further indicates the inorganic and organic parts are uniformly mixed.

The reversible photochromic response of the prepared composite fibers was also investigated upon continuous UV irradiation and infrared heating process as shown in Fig. 5a. The color of composite fiber changes from initial light yellow to dark blue under UV exposure within $7 \mathrm{~min}$, and gradually turns back to its initial status by using infrared lamp heating within about $2 \mathrm{~h}$. Fig. $5 \mathrm{~b}$ shows the reflectance spectrum of composite fiber as function of UV irradiation time. It's obvious that the reflective intensity remarkably decreased with continuous UV exposure, and reached saturation within $7 \mathrm{~min}$. The reflectance spectra of one single composite fiber was also recorded, as show in Fig. 5b, and the original unsmoothed spectral data was


Fig. 5 (a) Optical pictures of the prepared photochromic fiber show gradual color change under UV irradiation and infrared heating; (b) UVvis diffuse reflectance spectra of one single photochromic fiber with different UV irradiation time; (c) plots of the discoloration process for composite fiber in different environments by monitoring the reflection at $600 \mathrm{~nm}$ as a function of time; (d) the cyclic stability of the coloration-bleaching process of the prepared photochromic fiber by recording the reflectance intensity evolution at $600 \mathrm{~nm}$ for 5 cycles. shown in Fig. S6. $\dagger$ The discoloration process of composite fiber was also investigated by using various oxidation methods. Fig. S7 $\dagger$ shows the detailed experimental process, one single composite fiber colored with UV irradiation was evenly cut into four pieces, and each piece fiber was bleached with different method. As shown in Fig. 5c, under ambient conditions, the colored composite fiber can be oxidized slowly and gradually get back to original status in 2-3 days, while heating in oven or by infrared lamp at $100{ }^{\circ} \mathrm{C}$, the dark blue color can transfer to initial color in about $2 \mathrm{~h}$. If the sample is put in an ozone generator which can provide a large amount of oxygen radicals, the color will be bleached quickly in only 9-10 min. After comparing these methods, we chose the heating way via an infrared lamp as oxidation method to bleach the composite fiber's color because of its non-contact heating process and ozone free pollution. The color switching repeatability is essential for practical applications of photochromic fiber. The UV irradiation (within $7 \mathrm{~min}$ ) and infrared lamp heating (within $2 \mathrm{~h}$ ) were applied to study the photochromic reversibility and repeatability of composite fiber, and the corresponding reflectance intensities at $600 \mathrm{~nm}$ were recorded as 5 colorationbleaching cycles as shown in Fig. 5d. After 5 cycles, the color intensity only decreased slightly, which indicated the good reversibility of color turning.

The prepared photochromic fiber can be woven into some interesting shapes, such as grass ring, bow-tie, Chinese knot and even knitted fabric, as shown in Fig. 6a. And after UV irradiation, the color can change from initial light yellow to duck blue. The photochromic reversibility of the composite fiber may have a good prospect application in smart and functional textiles, which may be used in some special fields like military, firefighting, etc. Fig. $6 \mathrm{~b}$ shows the tensile strength-
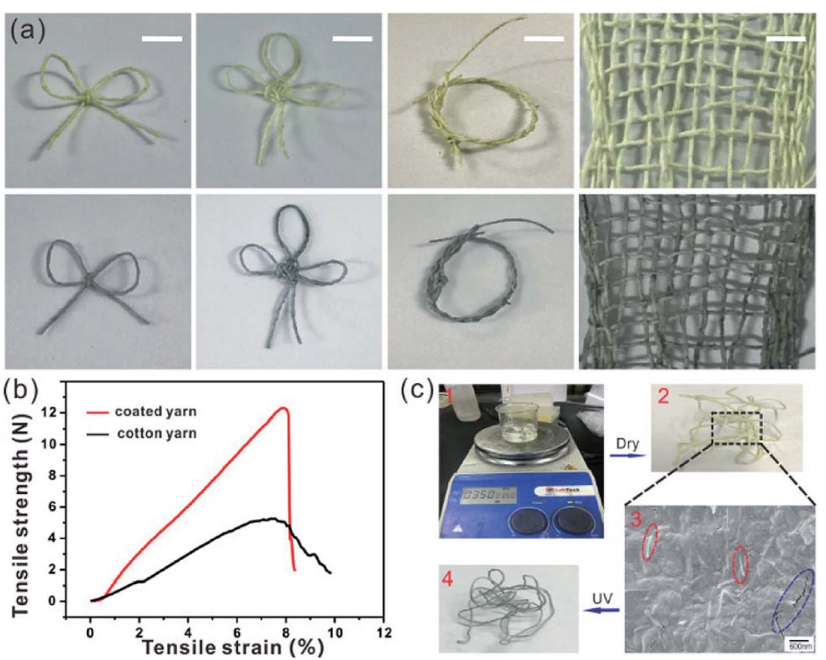

Fig. 6 (a) Pictures of the various shapes woven by prepared photochromic fiber before (upper) and after (below) UV irradiation, the scale bar is $1 \mathrm{~cm}$; (b) tensile strength-elongation curves for cotton and photochromic fiber; (c) the washability of prepared photochromic fiber, (1) picture of the simulated washing process by using a magnetic stirrer; (2) optical image of the washed photochromic fiber after drying; (3) SEM image of the surface of washed photochromic fiber; (4) the picture of the washed photochromic fiber after UV irradiation. 
elongation curves of prepared photochromic fiber and pure cotton fiber. Due to the composite coating layer with thickness of $4 \mu \mathrm{m}$ (drawing speed is $10 \mathrm{~mm} \mathrm{~min}^{-1}$ ), the photochromic fiber has a higher breaking strength of $12.2 \mathrm{~N}$, which is almost three times than that of cotton fiber. In order to investigate the washability of prepared photochromic fiber, a simulated washing process was performed by using magnetic stirrer as shown in Fig. 6c1. After washing at room temperature with $350 \mathrm{rpm}$ for $10 \mathrm{~min}$, the photochromic fiber's color (Fig. 6c2) was unchanged, which means the composite coating was not stripped from cotton fiber during the washing process. The SEM image in Fig. 6c3 further indicates the composite coating was not destroyed except for some cracks (area marked with blue dotted line). The washed composite fiber still has the photochromic property, as shown in Fig. 6c4, the fiber's color changed from light yellow into duck blue after UV irradiation. The wonderful mechanical property and washability indicate the prepared photochromic fiber may have good practicability.

\section{Conclusions}

In summary, $\mathrm{WO}_{3}$ nanorods were successfully synthesized by a simple one-step hydrothermal method, and the assynthesized $\mathrm{WO}_{3}$ nanorods were further uniformly dispersed in PVA solution to form homogeneous composite coating solution. The photochromic fiber was fabricated by coating the as-obtained composite solution onto the surface of cotton fiber. The prepared photochromic fiber shows fast photochromic rate and good reversible color change performance, wonderful mechanical strength and good washability, and the photochromic fiber can be woven into various patterns and fabrics. The $\mathrm{WO}_{3}$-based photochromic solution coating method may provide a step toward the development of photochromic fiber for functional textiles, which may have potential application in the areas of military camouflage, safety warning and entertainment, et al.

\section{Conflicts of interest}

There are no conflicts to declare.

\section{Acknowledgements}

This work is supported by the National Natural Science Foundation of China (NSFC) (No. 51603227 and 51603228), and National Key R\&D Program of China (Grant No. 2017YFE0112000).

\section{Notes and references}

1 C. Sun, W. Li and W. Chen, Sensors, 2017, 17, 1848.

2 I. Kim, K. Woo, Z. Zhong, P. Ko, Y. Jang, M. Jung, J. Jo, S. Kwon, S.-H. Lee, S. Lee, H. Youn and J. Moon, Nanoscale, 2018, 10, 7890.

3 M. d. Vos, R. Torah, M. G. Gostkiewicz and J. Tudor, J. Disp. Technol., 2016, 12, 1757.
4 P.-C. Hsu, X. Liu, C. Liu, X. Xie, H. R. Lee, A. J. Welch, T. Zhao and Y. Cui, Nano Lett., 2015, 15, 365.

5 N. Soin, T. H. Shah, S. C. Anand, J. Geng, W. Pornwannachai, P. Mandal, D. Reid, S. Sharma, R. L. Hadimani, D. V. Bayramol and E. Siores, Energy Environ. Sci., 2014, 7, 1670.

6 D. Liu, Y. Li, S. Zhao, A. Cao, C. Zhang, Z. Liu, Z. Bian, Z. Liu and C. Huang, RSC Adv., 2013, 3, 13720.

7 Z. Wang, J. Cheng, Q. Guan, H. Huang, Y. Li, J. Zhou, W. Ni, B. Wang, S. He and H. Peng, Nano Energy, 2018, 45, 210.

8 Q. Li, K. Li, H. Fan, C. Hou, Y. Li, Q. Zhang and H. Wang, J. Mater. Chem. C, 2017, 5, 11448.

9 Y. Zhang, Y. Zhao, J. Ren, W. Weng and H. Peng, Adv. Mater., 2016, 28, 4524.

10 Y. Shen, N. C. Harris, S. Skirlo, M. Prabhu, T. B. Jones, M. Hochberg, X. Sun, S. Zhao, H. Larochelle, D. Englund and M. Soljačić, Nat. Photonics, 2017, 11, 441.

11 B. Gauvreau, N. Guo, K. Schicker, K. Stoeffler, F. Boismenu, A. Ajji, R. Wingfield, C. Dubois and M. Skorobogatiy, Opt. Express, 2008, 16, 15677.

12 H. Peng, X. Sun, F. Cai, X. Chen, Y. Zhu, G. Liao, D. Chen, Q. Li, Y. Lu, Y. Zhu and Q. Jia, Nat. Nanotechnol., 2009, 4, 738.

13 F. M. Kelly and C. Cochrane, Color-Changing Textiles and Electrochromism, in Handbook of Smart Textiles, ed. X. Tao, Springer, Singapore, 2015.

14 L. v. d. Werff, I. L. Kyratzis, A. Robinson, R. Cranston, G. Peeters, M. O'Shea and L. Nichols, J. Mater. Sci., 2013, 48, 5005.

15 W. M. Kline, R. G. Lorenzini and G. A. Sotzing, Color. Technol., 2014, 130, 73.

16 J. Xu, W. Ji, C. Li, Y. Lv, Z. Qiu, L. Gao, E. Chen, J. W. Y. Lam, B. Tang and L. Jiang, Adv. Opt. Mater., 2018, 1701149.

17 J. Yao, A. A. Kaberniuk, L. Li, D. M. Shcherbakova, R. Zhang, L. Wang, G. Li, V. V. Verkhusha and L. V. Wang, Nat. Methods, 2016, 13, 67.

18 C. Ji, L. Ma, M. Yin, W. Yang and K. Pan, Chem.-Asian J., 2016, 11, 2316.

19 M. Trigo-López, J. L. Pablos, A. Muñoz, S. Ibeas, F. Serna, F. C. García and J. M. García, Polym. Chem., 2015, 6, 3110.

20 J. Zhang, S. He, L. Liu, G. Guan, X. Lu, X. Sun and H. Peng, J. Mater. Chem. C, 2016, 4, 2127.

21 G. Huang, L. Liu, R. Wang, J. Zhang, X. Sun and H. Peng, J. Mater. Chem. C, 2016, 4, 7589.

22 X. Lu, Z. Zhang, X. Sun, P. Chen, J. Zhang, H. Guo, Z. Shao and H. Peng, Chem. Sci., 2016, 7, 5113.

23 A. Laforgue, G. Rouget, S. Dubost, M. F. Champagne and L. Robitaille, ACS Appl. Mater. Interfaces, 2012, 4, 3163.

24 W. Qi, H. Li and L. Wu, J. Phys. Chem. B, 2008, 112, 8257.

25 S. Wang, W. Fan, Z. Liu, A. Yu and X. Jiang, J. Mater. Chem. C, 2018, 6, 191.

26 K. Kuboyama, K. Hara and K. Matsushige, Jpn. J. Appl. Phys., 1992, 31, 1609.

27 S. Yano, K. Kurita, K. Iwata, T. Furukawa and M. Kodomari, Polymer, 2003, 44, 3515.

28 W. Chen, H. Shen, X. Zhu, H. Yao and W. Wang, Ceram. Int., 2015, 41, 14008. 
29 S. Yamazaki, H. Ishida, D. Shimizu and K. Adachi, ACS Appl. Mater. Interfaces, 2015, 7, 26326.

30 Y. Zhou, A. Huang, S. Ji, H. Zhou, P. Jin and R. Li, Chem.Asian J., 2018, 13, 457.

31 R. Li, F. Hu, Q. Bao, S. Bao, Y. Qiao, S. Yu, J. Guo and C. M. Li, Chem. Commun., 2010, 46, 689.

32 D. Li, J. Wei, S. Dong, H. Li, Y. Xia, X. Jiao, T. Wang and D. Chen, ACS Appl. Mater. Interfaces, 2018, 10, 1701.

33 X. An, J. C. Yu, Y. Wang, Y. Hu, X. Yu and G. Zhang, J. Mater. Chem., 2012, 22, 8525.
34 O. Rezaee, H. M. Chenari, F. E. Ghodsi and H. Ziyadi, J. Alloys Compd., 2017, 690, 864.

35 C. Dulgerbaki, A. I. Komur, N. N. Maslakci, F. Kuralay and A. U. Oksuz, Opt. Mater., 2017, 70, 171.

36 C. Dulgerbaki, N. N. Maslakci, A. I. Komur and A. U. Oksuz, Electroanalysis, 2016, 28, 1873.

37 J. Wei, X. Jiao, T. Wang and D. Chen, ACS Appl. Mater. Interfaces, 2016, 8, 29713.

38 W. Yuan and K.-Q. Zhang, Langmuir, 2012, 28, 15418.

39 W. Yuan, N. Zhou, L. Shi and K.-Q. Zhang, ACS Appl. Mater. Interfaces, 2015, 7, 14064. 